\title{
Particle size analysis of niosomes as a function of temperature
}

\author{
E. I. Diskaeva ${ }^{1}$, O. V. Vecher ${ }^{1}$, I. A. Bazikov ${ }^{1}$, D. S. Vakalov ${ }^{2}$ \\ ${ }^{1}$ Stavropol State Medical University, Mira, 310, Stavropol, 355017, Russia \\ ${ }^{2}$ North Caucasus Federal University, Pushkina, 1, Stavropol, 355000, Russia \\ vecher.olga@mail.ru
}

PACS 61.46.-w, 65.80.tn, 87.16. Dg

DOI 10.17586/2220-8054-2018-9-2-290-294

The aim of the present investigation is to study and evaluate influence of temperature factor on the size of niosomes and particle size distribution. Particle size was defined with analysis electronic microscopy images. By the analysis of the probability density functions and cumulative distribution functions for particle size was confirmed influence temperature on their equivalent diameter. On the basis of the statistical analysis, we proved possibility of management niosomes size using the change temperature on the niosomes dispersion.

Keywords: niosomes dispersion, nonionic surfactant vesicles, particle size distribution.

Received: 11 November 2017

Revised: 23 November 2017, 25 December 2017

\section{Introduction}

One of the more promising directions for the development of modern pharmacology is perfection of methods which have already confirmed the efficiency of drug delivery. There are vesicular drug delivery system such as liposomes and niosomes [1-4]. Niosomal drug delivery systems have great advantage for poorly soluble drug by increasing its solubility, controlling its release and prolong its activity over period of time.

Niosomes are stable nonionic microscopic vesicles consisting of one or several membrane of various structures. Wide application of nonionic surfactants and lipids in designing of such systems is caused by their biocompability, ability, ability for biodegradation and also low toxicity.

Advantages noisome-delivered drugs include an in increase bioavailability of medical products by increased penetration through biological membrane, in propagation of pharmalogical action and selective accumulation in a place of entered [5-8]. Niosomes can be used for excipient biological active products, pharmaceuticals and non-pharmaceutical drugs.

Principles of a target drug delivery system used possibility management in wide range of niosomes size. For example, at oral - less than $1000000 \mathrm{~nm}$, at a local entered - less than $300 \mathrm{~nm}$, at enter in a circulation blood $10 \mathrm{~nm}[9]$.

Many methods, developed now give us possibility to change and controlled diameter niosomes, their physical and chemical properties, surface structure in a preparation process [10-12]. The particle size and particle distribution of niosomes have an important role on their biodistribution. A narrow particle size distribution is desired for drug carriers [13]. At the same time, there has been keen interest to change the size of already-prepared solutions using the temperature. Temperature change of niosomal system affects assembly of surfactants into vesicles and also induced vesicle shape transformations [14].

\section{Experimental}

In the present study niosomes were used that consisted of a shell in the form of a water-insoluble double layer of a nonionic surfactant, which is a group of dimethiconecopolyol substances that are esters of polyethylene glycol and polydimethylsiloxane (PDMS) backbone [15-17].

To obtain the silicone-based capsules, we used physico-chemical methods for the synthesis of molecules. The shell of the obtained niosome vesicles were generated from PEG-12 Dimethicone. In the hydrophilic part of dimethicone there are functional groups of silicon oxide. The length of the Si-O bond was $1.6 \AA$, which is much longer than the C-C bond of $1.4 \AA$. Due to this, the functional groups of the molecules are able to rotate with respect to each other. This provided niosomes with greater elasticity than liposomes made up of phospholipids. The use of PEG-12 Dimethicone promoted the formation of vesicles without significant energy effort. The $\mathrm{Si}-\mathrm{O}-\mathrm{Si}$ bond angle was $130^{\circ}$, in contrast to the $109^{\circ}$ of the C-C-C bond, which increased the elasticity and stability [18]. The stage of vesicle formation occurred with intensive mechanical mixing of the mixture using an automatic reclosure homogenizer. The dispersion was then placed in a vessel for ultrasonic treatment. Ultrasonic irradiation 
was carried out at a frequency of $20 \mathrm{kHz}$; power - $200 \mathrm{~W}$, exposure time - 10 minutes. Monolamellar niosomes were formed. Then, samples were diluted with ultrapure water $(\mathrm{C}=0.01 \%)$ and heating on the water bath (Ulab, China) on temperature interval 303-333 K.

The dispersion of nanoparticles was studied using scanning electron microscopic method (SEM Tescan Mira $3 \mathrm{Im})$. The particle size determined using computer program Image J.Excel statistical package program was used to perform the analysis. The measurements were carried out after 1.5 hours and after 7 and 14 days.

The image in Fig. 1 presents come series of microscopy pictures. Apparently the water solution of niosomes consists of particles with various diameters.

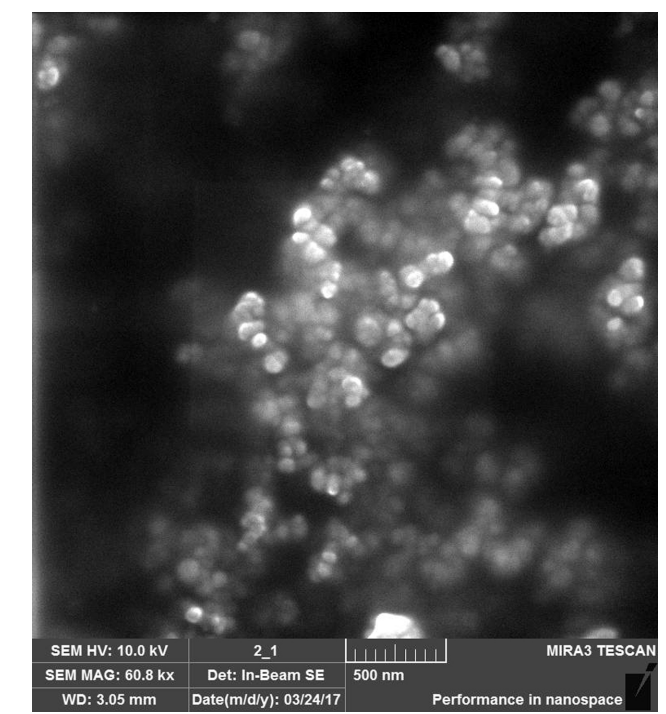

$\mathbf{a}$

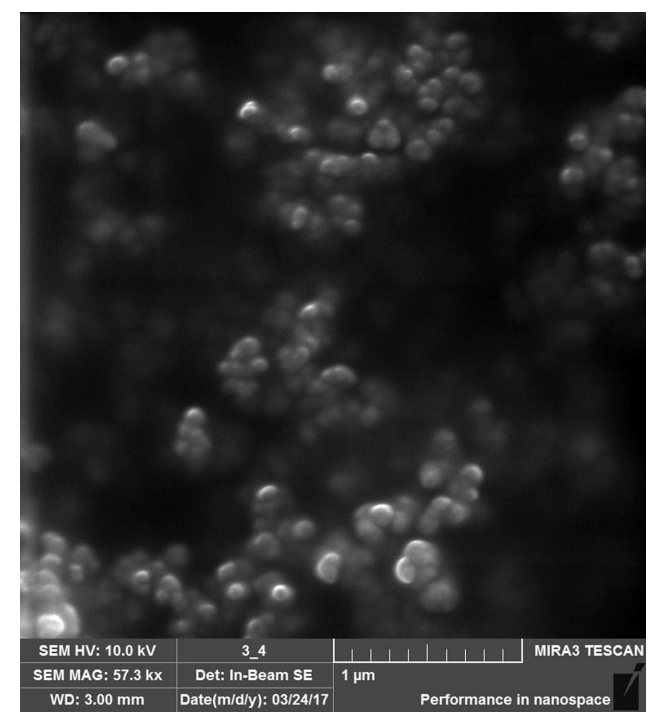

b

FIG. 1. Scanning electron microscopy (SEM) micrographs of niosomes (a - after $1.5 \mathrm{~h}$; b - after 14 days)

As can be seen from the presented microphotographs, niosomes, for the most part, are spherical particles. This means that to determine the equivalent diameter of particles, we can use the projected diameter. The area of the projected diameter is equal to the area of the image of the projection of the particle.

Since the area of the projection of a spherical particle is:

$$
S_{P}=\frac{\pi \delta_{P}^{2}}{4}
$$

Then, the average projection diameter calculation is:

$$
\delta_{P}=\sqrt{\frac{4 S_{P}}{\pi}} .
$$

To obtain information on the particle size for each temperature regime, fractions with a total number of a part of 500 was investigated. This procedure was done in triplicate for each sample.

The cumulative distribution function, $D(\delta)$, was used to evaluate the average particle size. This function is equal to the ratio of all particles, whose diameter does not exceed the value $\delta$, to the total number of particles expressed in fractions or percentages (Fig. 2).

For a more detailed analysis, can use the function obtained by differentiating the cumulative distribution function, i.e. distribution density:

$$
f(\delta)=\frac{d D(\delta)}{d \delta}
$$

In this case, the following equation must be true:

$$
\int_{\delta_{\min }}^{\delta_{\max }} f(\delta) d \delta=D\left(\delta_{\max }\right)-D\left(\delta_{\min }\right) .
$$




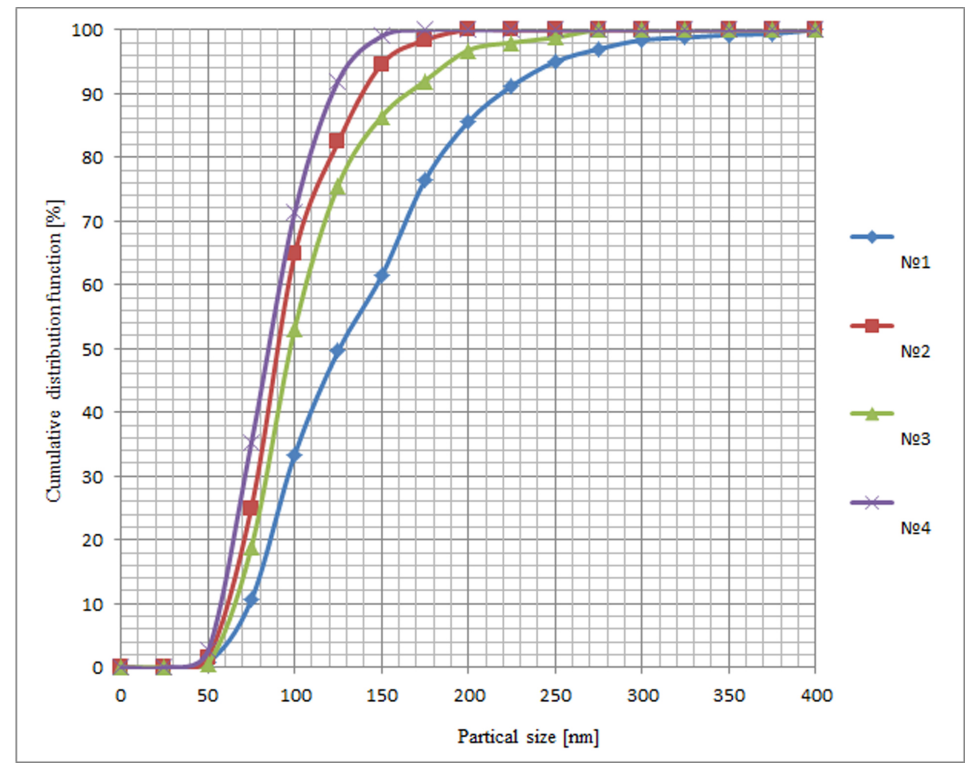

FIG. 2. Cumulative distribution function of particle size in niosomal dispersions after heating to temperatures of $303 \mathrm{~K}$ (No 1), $313 \mathrm{~K}$ (No 2), $323 \mathrm{~K}$ (No 3) and $333 \mathrm{~K}$ (No 4)
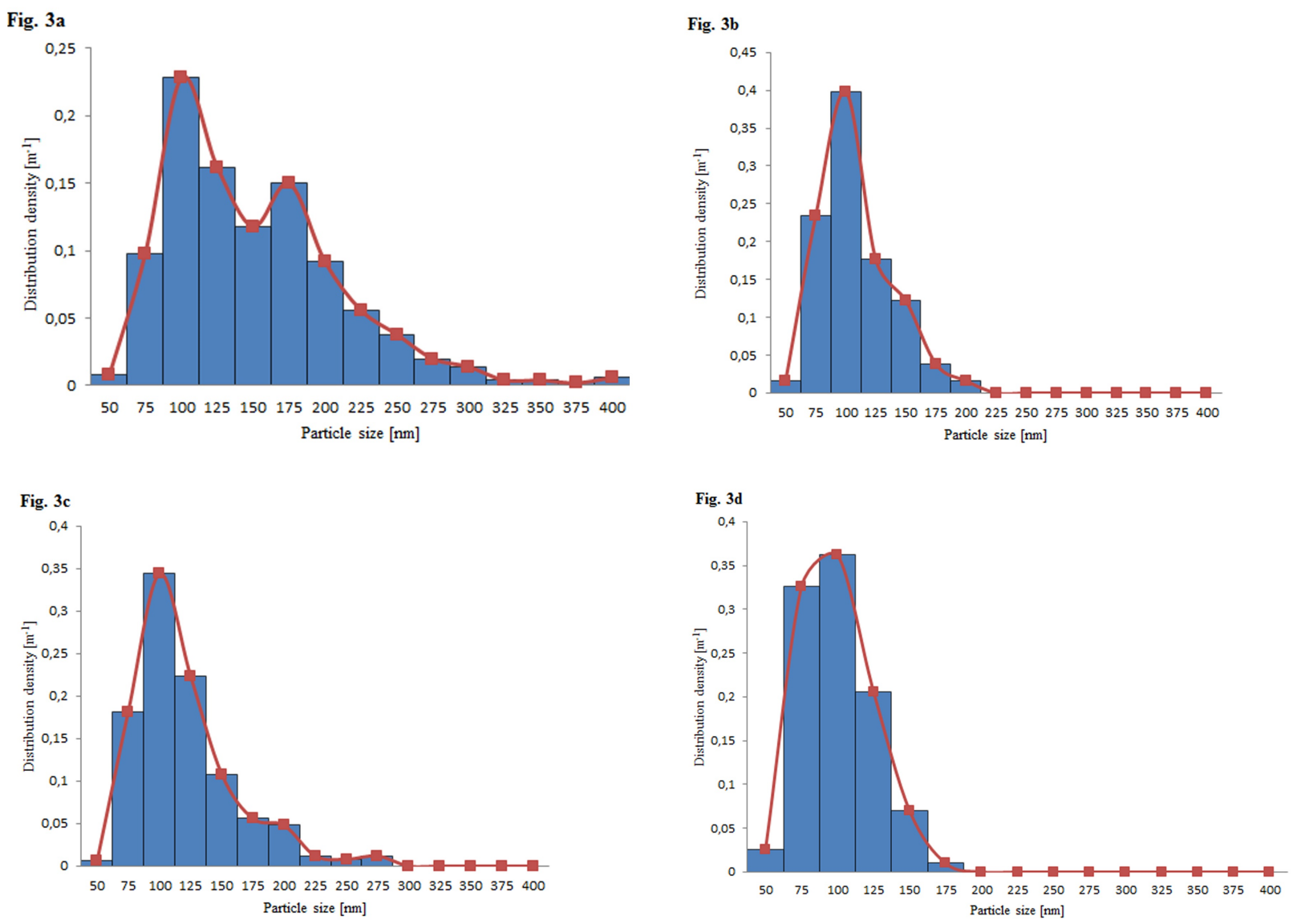

FIG. 3. Column charts of particle size distribution in niosomal dispersions subjected to heating to temperatures of $303 \mathrm{~K}(\mathrm{a}), 313 \mathrm{~K}(\mathrm{~b}), 323 \mathrm{~K}$ (c), and $333 \mathrm{~K}$ (d) 
Experimental studies of the composition of niosomal dispersion were accompanied by the construction of the function in the form of a column charts and completed with mathematical approximation. The column charts of the particle size distribution for different temperature regimes are shown on Fig. 3.

The area of each rectangle is the percentage of the fraction with a defined particle size interval. The correctness of the approximation was controlled by the equality:

$$
\sum_{i=1}^{k} f_{i} \Delta \delta_{i}=\int_{0}^{\infty} f(\delta) d \delta=1 .
$$

Probability density function of particles by the size subjected to heating the different temperatures $303-333 \mathrm{~K}$ accordingly are shown on Fig. 4.

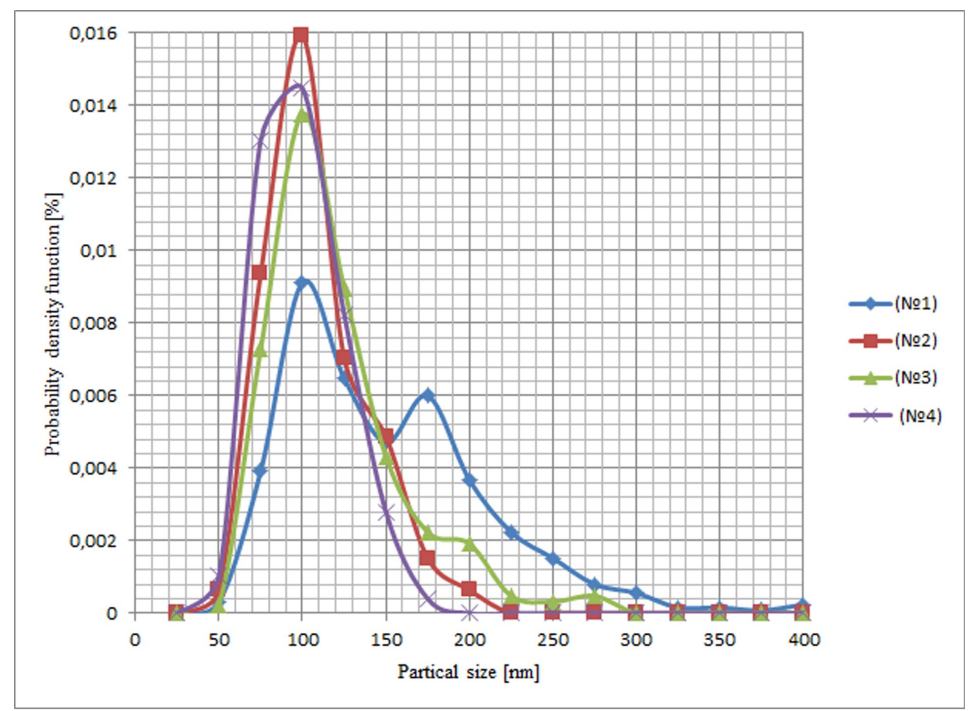

FIG. 4. Probability density function of particle size distribution in niosomal dispersions subjected to heating to temperatures of $303 \mathrm{~K}$ (No 1), $313 \mathrm{~K}$ (No 2), $323 \mathrm{~K}$ (No 3) and $333 \mathrm{~K}$ (No 4)

\section{Results and discussion}

Figure 4 shows that the probability density functions presented monomodal distribution and bimodal particle size distribution. Using these curves, we can determine possible value of the equivalent diameter corresponding to the maximum number of particles in niosomal dispersion, i.e. the most likely size of vesicles. Comparison of these values for different temperature regimes demonstrates homogenization with respect to particle size of niosomes dispersion.

The size of niosomes is of enormous importance for transporting drugs and their therapeutic effectiveness. So, if the niosomes diameter decreases, then the efficiency of penetration into deep layers of a skin is increased. Therefore, the most interesting are monolamellar niosomes, with diameter approximately 80-100 nm, having a thin flexible shell. These niosomes move more efficiently through intercellular spaces, lymphatic and blood capillaries. Dimethicone, which forms a bilayer structure in an aqueous medium, made it possible to obtain a hyperfine and elastic membrane niosomes.

Considering the interval of values of the vesicles diameter in the range from $75 \mathrm{~nm}$ to $125 \mathrm{~nm}$, it can be seen that the effect of temperature increase has a direct effect on the decrease of particle size: from $48.8 \%$ at $303 \mathrm{~K}$ to $89.4 \%$ at $333 \mathrm{~K}$. Thus, on the basis of the statistical analysis, we have proved the possibility of controlling niosome size using the temperature regimes, acted on the niosomes dispersion. Heat energy, in this case, is expended an endothermic phase transitions and the process destruction of conglomerates or temporary associates of niosomes.

The aqueous suspension of niosome is a colloidal system, and the interaction between the particles can lead to the formation of either stable aggregates or temporary associates due to coagulation and flocculation. As a result of these processes, niosomal dispersion is a combination of structures of the following types: small individual vesicles, flocculated vesicles, aggregated vesicles and fused vesicles.

Niosomes of small dimensions are thermodynamically unstable, so their content is detected only after a short time after the preparation of the suspension, provided it has a small concentration. The distance between niosomes 
in the aggregates is so small that a strong intervesicular interaction arises between them. This is accompanied by a curvature of the contacting surfaces and further leads to the formation of more profitable energetically large niosomes, i.e. they are merged. With the rise in temperature, the fusion of niosomes in aggregates slows down under the influence of the forces of repulsion of the structuredness of water in the space between them and the increase in Brownian motion.

\section{Conclusions}

Thus, an analysis of the effect of heating niosomal dispersion on the average particle size reveals a tendency to decrease the diameter of niosomes with increasing temperature. Niosomes subjected to heat treatment become more stable in time and retain an average particle size of about $100 \mathrm{~nm}$. This approach can be recommended for further improvement of methods of preparation and storage of stable drug forms.

\section{References}

[1] Growi R., Balaji P., Vijayalakshmi P., Preethy Mol.G., Karthik Raja P. Niosomes - a vesicular drug delivery system. International Journal of Current Research. 2013, 5(08), P. 2239-2244.

[2] Viviane F. Naggar, Safaa S. Elgamal, Ahmed N. Allam Formulation and Physicochemical Characterization. Journal of American Science, 2012, 8(9), P. 417-428.

[3] Abhinav Kumar, Pal Jogender Lal, Jaiswal Amit, Singh Vishwabban. Review of niosomes as novel druid delivery system. Int. Research Journal of Pharmacy, 2(5) 2011, P. 61-65.

[4] Zerrin Sezgin Bayindir, Nilufer Yuksel. Characterization of niosomes Prepared with various Nonionic Surfactants for paclitaxel Oral delivery. Journal of Pharmaceutical sciences, 2010, 99(4), P. 2049-2060.

[5] Bazikov I.A. A method for transdermal transfer of active substances using niosomes on the basis of PEG-12 dimethicone. RF patent 2539396, 2014.

[6] Bazikov I.A., Zelensky V.A., Gukasyan A.L. Nanobiotechnologies of the use of niosomes in dentistry, Maikop: Quality, 2015, 112 p.

[7] Malay K. Das, Narahari N. Palei. Sorbitan ester niosomes for topical delivery of rofecoxib. Indian Journal of Experimental Biology, 2011, 49, P. 438-445.

[8] Sakthivel M., Kannan K., Manavalan R., Senthamarai R. Non Ionic Surfactant Vesicles. A Review. Research Journal Pharmaceutical, Biological and Chemical, 2012, 3(01), P. 604-614.

[9] Handbook of nanostructured materials and nanotechnology. Ed. H.S. Nalwa. Boston: Academic Press, 2000,3461 p.

[10] Gannu P. Kumar, Pogaku Rajieshwarrao. Nonionic Surfactants vesicular system for effective druid delivery - an overview. Acta Pharmaceutica Simca B, 2011, 1(4), P. 208-219.

[11] Apeora Agarwal, Weha Jugal, Gauree Kukreti, Sachdew Yadav. Niosomes of targeted druid delivery systems. International Journal of Pharmaceutical Science Review and Research, 2012, 12(1), P. 53-60.

[12] Ahuja N., Saini V., Bishnoi V.K., Garg A., Hisoria M., Sharma J., et al. Formulation and evaluation of lansoprazol noisome. Rasayan J. Chem., 2008, 1, P. 561-564.

[13] Zerrin Sezgin Bayindir, Nilufer Yuksel. Investigation of formulation variables and excipient interaction on the production of niosomes. Pharm. Sci. Tech., 2012, 13, P. 826-835.

[14] Arunothayanum P. et al. Extrusion of niosomes from capillaries approaches to a pulsed delivery device. J. Cont Release, 1999,60 , P. 1999.

[15] Bazikov I.A., Beyer E.V., Maltsev A.N., et al. Investigation of the cardiotoxicity of the niosomal form of doxorubicin. Medical Gazette of the North Caucasus, 2016, 11(3), P. 421-424.

[16] Bazikov I.A., Lukinova V.V., Maltsev A.N., et al. The interaction of niosomal doxorubicin with cell membranes. Medical Gazette of the North Caucasus, 2016, 11(1), P. 108-110.

[17] Bazikov I.A., Lukinova V.V., Malinina N.I., Maltsev A.N. Study of the mechanisms of intercellular interaction of the niosomal form of the antitumor drug doxorubicin with plasma membranes. The Eurasian Union of Scientists, 2016, 3(24), P. 34.

[18] Bazikov I.A., Omelyanchuk P.A. The method of delivery of biologically active substances with the help of niosomes RF patent 2320323 , 2008. 